Arq. Bras. Med. Vet. Zootec., v.64, n.4, p.891-898, 2012

\title{
Mordedura canina e atendimento antirrábico humano em Minas Gerais
}

\author{
[Dog bite and human anti-rabies healthcare, Minas Gerais] \\ V.M.R. Oliveira ${ }^{1}$, P.L.L. Pereira ${ }^{2}$, J.A. Silva ${ }^{2}$, C.F.J. Miranda ${ }^{3}$, K.O. Rodrigues ${ }^{4}$, \\ T.O. Rodrigues ${ }^{5}$, E.C. Moreira ${ }^{2}$ \\ ${ }^{1}$ Faculdade de Medicina - Universidade Federal de Minas Gerais - Belo Horizonte, MG \\ ${ }^{2}$ Escola de Veterinária - Universidade Federal de Minas Gerais - Belo Horizonte, MG \\ ${ }^{3}$ Ministério da Saúde - Brasília, DF \\ ${ }^{4}$ Hospital Felício Rocho - Belo Horizonte, MG \\ ${ }^{5}$ Faculdade de Ciências Médicas de Minas Gerais - Belo Horizonte, MG
}

\begin{abstract}
RESUMO
O objetivo do presente trabalho foi descrever e avaliar a mordedura canina e o atendimento antirrábico humano em Minas Gerais, de 1999 a 2004, correlacionando fontes de informação e áreas de risco predeterminadas para raiva humana transmitida por cão. Realizou-se um estudo observacional descritivo retrospectivo, utilizando-se, de forma adaptada, a análise exploratória de prontuários dos atendimentos da Superintendência de Epidemiologia da Secretaria de Estado da Saúde de Minas Gerais (339.012 de atendimentos), do Sistema de Informação de Notificação de Agravos, do Sistema de Informações sobre Mortalidade, do Sistema de Informações Hospitalares e do Programa Nacional de Imunizações (132.452 fichas). Para a classificação dos agravos, usou-se o Código Internacional de Doenças (10 a revisão). Os dados foram armazenados e analisados com auxílio dos softwares Epi-Info, Tab-Win e Office ${ }^{\circledR}$. Verificou-se que o tratamento antirrábico humano é excessivo nas áreas de baixo e médio risco para raiva e, ao contrário, reduzido nas áreas de alto risco. O perfil do paciente é estudante masculino, menor de 14 anos, residente em área urbana de baixo risco para raiva humana transmitida por cão, com mordedura única nos membros, provocada por cão sadio e observável. Os sistemas de informação não oferecem a confiabilidade necessária ao médico responsável para a prescrição do tratamento antirrábico adequado. A profilaxia da raiva deve ter um aspecto multicêntrico, com interfaces na atenção tanto à saúde humana quanto à animal, o que não tem ocorrido, propiciando falhas na vigilância e no atendimento do agravo.
\end{abstract}

Palavras-chave: raiva, atendimento antirrábico, vigilância, mordedura canina

\begin{abstract}
The objective of the present paper is describe and evaluate dog bite and some aspects of anti-rabid human care in Minas Gerais during five years, correlating the sources of information and epidemiological risk areas defined for human rabies transmitted by dogs in the State. We performed an observational retrospective study by adapted exploration form analysis, from 1999 to 2004, using databases of Epidemiology of Minas Gerais and National Information Systems of reportable disease, Immunization, Mortality, Hospitalization, International Code of Diseases (10th revision). The areas of risk for human rabies transmitted by dogs were pre determined. The dog bite is still the main complaint that leads to care. The profile of the patient is a male student, under 14 years of age, with a single wound in members, resident in an urban low risk area for human rabies transmitted by dogs, and is healthy and observable. The treatment is excessive in areas of low and medium risk. In high-risk areas, there is a low indication of treatment. Information systems do not offer the reliability required by the doctor responsible for prescribing the appropriate anti-rabies treatment. Rabies prophylaxis should have a multi-centre aspect, with interfaces in attention to health and veterinary, which has not occurred, providing surveillance failures.
\end{abstract}

Keywords: rabies, anti-rabies care, surveillance, dog bite

Recebido em 7 de março de 2012

Aceito em 2 de agosto de 2012

E-mail:valmro@uol.com.br 


\section{INTRODUÇÃO}

A raiva canina limita-se quase exclusivamente aos países em desenvolvimento. Estima-se que, nesses países, de 40 a 70 mil pessoas morrem depois de terem sido mordidas por cães raivosos, e cerca de 10 milhões recebem tratamento pósexposição para raiva por ano (World..., 2001). Grande parte do problema deve-se à incompreensão do papel do cão na sociedade e à falta de padronização e aplicação da informação nos sistemas de controle da raiva canina, o que não ocorre nos países desenvolvidos.

No Brasil, a vigilância em saúde e a imunização tanto humana quanto animal são bases para o programa de controle do agravo. Porém, os dados epidemiológicos gerados não são processados, avaliados, configurados e transmitidos em tempo hábil aos componentes dos sistemas para as tomadas de decisão adequadas. Essas informações são essenciais tanto para os médicos, que devem decidir sobre a administração do tratamento pós-exposição, como para os veterinários, que devem tomar as medidas sanitárias adequadas a respeito do animal agressor.

Existem esquemas de tratamento diferenciados e normatizados pelo Ministério da Saúde (MS) que variam de acordo com a existência de tratamento anterior, características do ferimento, espécie e estado clínico do animal agressor e atividade profissional do paciente, mas não consideram a situação epidemiológica local. Por sua vez, a cobertura vacinal canina tem-se mantido acima de $80 \%$, segundo a estimativa populacional canina indicada pelo MS. Porém, essa população canina é, em geral, subestimada, pois os resultados obtidos em campanhas de vacinação antirrábica animal em diversos municípios do estado superam, muitas vezes, mais de $100 \%$ de cães vacinados.

O tratamento antirrábico pós-exposição vem aumentando substancialmente, tendo-se duplicado a indicação de tratamento nos últimos 10 anos. O tipo e a gravidade de exposição ao vírus rábico têm sido negligenciados pelas autoridades sanitárias, o que superestima a indicação de tratamento, independentemente de sua efetiva necessidade. Em geral, os tratamentos pós-exposição no estado permanecem em níveis elevados e caros.
Assim, esta pesquisa descreve e avalia alguns aspectos para a saúde pública da mordedura canina e do atendimento antirrábico humano em Minas Gerais, de 1999 a 2004, período em que foi implantado e implementado o Sistema Nacional de Informação de Agravos de Notificação (SINAN) para este atendimento, juntamente com as informações da Secretaria de Saúde do Estado de Minas Gerais. Assim, correlacionaram-se as fontes de notificação existentes com as áreas de risco epidemiológico definidas para raiva humana transmitida por cão no estado e a interface com os outros componentes do sistema de informação no Sistema Único de Saúde - SUS.

\section{MATERIAL E MÉTODOS}

O presente estudo foi feito no estado de Minas Gerais, cuja área é de $586.552,4 \mathrm{~km}^{2}$, com 17.891.494 habitantes, residentes em 853 municípios (IBGE, 2000). O Estado possui 28 Diretorias Descentralizadas de Saúde (DAD) da Secretaria de Estado da Saúde de Minas Gerais (SES/MG).

Realizou-se estudo observacional descritivo retrospectivo, de 1999 a 2004, em Minas Gerais, com base nos registros de raiva humana, de encefalites não especificadas e demais agravos relacionados, segundo o Código Internacional de Doenças, $10^{\mathrm{a}}$ revisão - CID-10 (CID A82 Raiva humana, CID A86 - Encefalites não especificadas, CID W54 - Mordedura ou golpe proferido por cão, CID W55 - Agressão por outros mamíferos). Foram utilizados os seguintes bancos de dados: Sistema de Informação de Agravos de Notificação (SINAN), Programa Nacional de Imunizações - PNI, Sistema de Informações sobre Mortalidade (SIM), Sistema de Informações Hospitalares - SIH, Superintendência de Epidemiologia da SES/MG (planilhas de dados provenientes dos serviços municipais de atendimento antirrábico, consolidados nas DADs pela Coordenadoria de Zoonoses - ZOO, dados de raiva animal e de eventos adversos à vacina antirrábica humana do PNI). A informação colecionada foi analisada de acordo com o método de análise exploratória de prontuários adaptado do Centers of Disease Control and Prevention - CDC (Center..., 2004).

Para a caracterização e a análise do perfil do atendimento antirrábico humano, dos 
coeficientes de incidência de agressões e dos tratamentos prescritos por área de risco, consideraram-se, nas fichas do SINAN e nas planilhas da ZOO, as seguintes informações: identificação do atendimento segundo o número da ficha, data e local da ocorrência, residência, ocupação e data de nascimento do agredido; tipo de exposição ao vírus rábico, localização e tipo do ferimento; espécie, condição, modo de ocorrência de agressão e diagnóstico clínico e laboratorial do animal; tratamento de acordo com o número de doses e o tipo de vacina, antecedentes de tratamento antirrábico, indicação de soro antirrábico, reações adversas e dados sobre a interrupção do tratamento.

Utilizou-se a classificação das áreas de risco para raiva humana transmitida por cão determinada por Miranda et al. (2003), que caracterizaram o estado em áreas de risco nulo, baixo, médio e alto. As áreas de risco nulo e baixo risco foram agregadas, por possuírem indicações equivalentes para o atendimento e o tratamento antirrábico humano, quando considerado o risco epidemiológico na prescrição do tratamento pósexposição.

Os dados obtidos das planilhas da Coordenadoria de Zoonoses da SES-MG - ZOO foram armazenados e analisados, utilizando-se o programa Epi Info 6.04 ((Dean et al., 1995). Os dados do SINAN foram analisados com auxílio do Programa Tabwin ${ }^{\circledR}$. As tabelas e os gráficos foram elaborados usando-se o programa Excel ${ }^{\circledR}$.
As análises de tendência foram feitas pelo método dos mínimos quadrados (Sampaio, 1998).

\section{RESULTADOS E DISCUSSÃO}

$\mathrm{O}$ atendimento antirrábico humano é feito em pessoas que se julgam expostas ao vírus rábico, por demanda espontânea ou de seu responsável, no caso de crianças, idosos e pacientes com necessidades especiais. $\mathrm{O}$ número total de atendimentos avaliados foi 132.452 do SINAN e 339.012 da ZOO, segundo o local do registro. O coeficiente geral de atendidos no período foi de 3,11 por 1.000 habitantes, segundo os dados da ZOO (Tab. 1). O coeficiente de tratados/atendidos na ZOO foi de 2,28 por 1.000 habitantes, e a proporção de tratados/atendidos foi de 73,27\% no período (Tab. 1). O coeficiente de pacientes atendidos no SINAN foi de 1,22 por 1.000 habitantes (Tab. 1), considerando-se, neste caso, que há subnotificação nos primeiros anos de implantação do Programa do SINAN, em razão das dificuldades inerentes à operacionalização do sistema no Estado. Não houve notificações no SINAN de pacientes atendidos que não receberam imunobiológicos, $\mathrm{o}$ que não permite a avaliação entre atendidos/tratados e demais análises que necessitem dessa informação. A frequência de registros do SINAN aumentou gradativamente ao longo do período de estudo, equivalendo-se ao da ZOO em 2004 (Tab. 1).

Tabela 1. Pacientes atendidos e que receberam tratamento antirrábico, segundo dados da ZOO e do SINAN, Minas Gerais, 1999 a 2004

\begin{tabular}{|c|c|c|c|c|c|c|c|c|}
\hline Ano & $\begin{array}{c}\text { Pacientes } \\
\text { atendidos } \\
\mathrm{ZOO}\end{array}$ & $\begin{array}{c}\text { Pacientes } \\
\text { tratados } \\
\mathrm{ZOO}\end{array}$ & $\begin{array}{l}\text { Pacientes } \\
\text { atendidos } \\
\text { SINAN }\end{array}$ & $\begin{array}{c}\text { População } \\
\text { Total }\end{array}$ & $\begin{array}{l}\text { Coeficiente } \\
\text { paciente } \\
\text { atendido } \\
\mathrm{ZOO} \text { por } \\
1.000 \mathrm{hab}\end{array}$ & $\begin{array}{l}\text { Coeficiente } \\
\text { paciente } \\
\text { tratado } \\
\mathrm{ZOO} \text { por } \\
1.000 \mathrm{hab}\end{array}$ & $\begin{array}{c}\text { Razão } \\
\text { tratado/ } \\
\text { atendido } \\
\mathrm{ZOO} \\
\%\end{array}$ & $\begin{array}{l}\text { Coeficiente } \\
\text { paciente } \\
\text { atendido } \\
\text { SINAN por } \\
1.000 \text { hab }\end{array}$ \\
\hline 1999 & 55.708 & 39.523 & 910 & 17.268 .278 & 3,23 & 2,29 & 70,95 & 0,05 \\
\hline 2000 & 52.038 & 38.010 & 3.038 & 17.891 .494 & 2,91 & 2,12 & 73,04 & 0,17 \\
\hline 2001 & 54.952 & 40.133 & 20.625 & 18.127 .024 & 3,03 & 2,21 & 73,03 & 1,14 \\
\hline 2002 & 58.894 & 42.275 & 30.865 & 18.343 .518 & 3,21 & 2,30 & 71,78 & 1,68 \\
\hline 2003 & 67.148 & 49.022 & 37.494 & 18.553 .335 & 3,62 & 2,64 & 73,01 & 2,02 \\
\hline 2004 & 50.272 & 39.438 & 39.520 & 18.762 .405 & 2,68 & 2,10 & 78,45 & 2,11 \\
\hline Total & 339.012 & 248.401 & 132.452 & 108.946 .054 & 3,11 & 2,28 & 73,27 & 1,22 \\
\hline
\end{tabular}

De modo geral, observou-se uma tendência de aumento na frequência dos tratamentos antirrábicos, demonstrada pelas duas fontes notificadoras. Porém, deve-se considerar que ainda há áreas silenciosas no Estado, pois apenas nove das 28 DADs apresentaram $100 \%$ de municípios com notificação. Nas demais DADs, há municípios com ausência de notificação durante o período do estudo.

Ao se considerar o coeficiente de atendimentos em 3,11 por 1.000 habitantes na ZOO e 1,22 no 
SINAN, estima-se uma subnotificação de pacientes em relação à população de Minas Gerais. Ressalta-se que, no período de estudo, não houve registros no SINAN de atendimentos no Centro de Saúde Carlos Chagas, tradicional referência para tratamento antirrábico do estado, sob administração da Secretaria Municipal de Saúde de Belo Horizonte.

Em relação à faixa etária, observou-se que há maior concentração de notificações em crianças e idosos, com ferimentos, sobretudo, nas extremidades do corpo. O maior número de notificações ocorreu em crianças até 14 anos de idade. Esta informação, no entanto, só pode ser avaliada a partir do banco de dados do SINAN, pois nas planilhas da ZOO não havia registros de agrupamento de faixa etária dos pacientes nas notificações. A maior frequência em crianças até a primeira fase da adolescência também foi relatada por Moreira et al. (1976), Garcia et al. (1999), (World..., 2001), France (2002) e Miranda et al. (2004). Verificou-se, ainda, que a curva de atendimento por faixa etária se manteve mesmo quando os dados foram ponderados por ano de notificação, indicando uma constância na proporcionalidade da ocorrência do agravo, excetuando os anos de 1999 e 2000, quando foi implantado o registro de notificações neste sistema (Fig. 1).

Ponderando as faixas etárias por gênero, constatou-se a maior frequência em crianças do sexo masculino até a pré-adolescência, o que também se verifica em outros países (World..., 2001). A distribuição do agravo entre os gêneros foi semelhante a partir da segunda metade da adolescência até a faixa etária menor de 40 anos, quando ocorreu um ligeiro predomínio nas notificações do sexo feminino, também observado por Miranda et al. (2004). Na frequência geral dos atendimentos, no período, predominou o sexo masculino $(60 \%)$ em relação ao feminino (40\%), fato este já relatado por Moreira et al. (1976) e por France (2002).

Em relação ao mês de ocorrência da agressão, há uma distribuição regular durante todos os meses do ano, com apenas uma ligeira elevação do número de casos entre os meses de julho e setembro, porém este período coincide com as campanhas de vacinação em massa contra a raiva canina e a felina. Nos dados da ZOO, observou-se ainda um aumento na frequência de notificações no mês de janeiro, período de férias escolares, o que também foi verificado em Belo Horizonte por Maurelli (2001).

De acordo com o local do ferimento no agredido, observou-se que, em crianças menores de 10 anos, há uma equivalência entre os percentuais de agressão em cabeça/pescoço, com $24 \%$ das ocorrências, membros inferiores (MMII) com $26 \%$ e agrupamento mão/pé com 23\%. Os membros superiores (MMSS) estão registrados em $16 \%$ das agressões, e o tronco e as mucosas em $8 \%$ e $4 \%$, respectivamente. Nos maiores de 10 anos, as agressões mais frequentes ocorreram no agrupamento mão/pé em $37 \%$ dos registros e nos MMII em 35\%. Nas demais regiões, registraram-se $8 \%$ de agressões nos MMSS, $4 \%$ no tronco e cabeça/pescoço e $1 \%$ em contato com mucosas.

Os locais das lesões mais frequentes em menores de 10 anos correspondem aos de maior inervação e mais próximos ao sistema nervoso central. Nesse tipo de exposição, havendo probabilidade da introdução do vírus, aumenta-se a urgência do início do tratamento antirrábico, pois o período de incubação da doença será mais curto, segundo a (World..., 2001).

A divisão anatômica proposta pela ficha de atendimento antirrábico do SINAN não permitiu a comparação com outras referências internacionais e nacionais encontradas, excetuando-se o agrupamento cabeça/pescoço e tronco. $\mathrm{Na}$ divisão anatômica das fichas, foi criado o agrupamento mãos/pés, inexistente nos outros sistemas.

Nos resultados encontrados, verificou-se que, aproximadamente, $42 \%$ das lesões na cabeça/ pescoço e $30 \%$ no tronco e lambeduras de mucosas ocorreram em crianças com até cinco anos de idade. Esses resultados são semelhantes aos encontrados por Amato Neto e Costa (2000) e pelo CDC (Center..., 2003). Nos EUA, segundo o CDC (Center..., 2003), 45,3\% das agressões por cão ocorreram nos MMII, 25,8\% nos MMSS, $22,8 \%$ na cabeça/pescoço e $6,0 \%$ no tronco.

Confirmando a tendência histórica, o principal animal agressor neste estudo foi o cão, seguido do gato e dos quirópteros. Na ficha do SINAN, não existe um campo específico para o registro de agressões por herbívoros, apesar de haver 
notificações de 11.277 atendimentos de acidentes com estes animais no banco da ZOO, no mesmo período de estudo. Além do mais, $75 \%$ dos municípios mineiros apresentam casos de raiva em herbívoros, conforme relatado por Silva et al. (2001). Por outro lado, nas fichas, encontrou-se local destinado ao preenchimento de agressões por raposas e primatas não humanos, animais que apresentam relevância epidemiológica para o Nordeste brasileiro (Araújo, 2000). Com relação aos quirópteros, as notificações nas quais aparecem como animais agressores corresponderam a $0,5 \%$ do total de registros no período de estudo.

Um fator que influencia o esquema de tratamento a ser adotado é o número de ferimentos produzidos pela agressão. As agressões são classificadas em únicas ou múltiplas, superficiais, profundas ou dilacerantes, conforme encontradas nas notificações do SINAN. Essa classificação não se encontra disponível nos registros do banco da $\mathrm{ZOO}$.

O estado clínico do animal agressor é informado pelo paciente ou seu acompanhante, normalmente leigos e com avaliação subjetiva. Este dado é fundamental na escolha do tratamento prescrito e só é válido para agressões por cão ou gato. Entretanto, essa informação não é cotejada como risco epidemiológico para a área onde ocorreu a agressão.

A situação no momento da agressão também influencia a prescrição do tratamento antirrábico. Porém, mesmo entre as áreas de alto risco, há diferenças de percepção do risco, como foi relatado por Belotto (2000). A maioria das agressões encontradas no presente estudo ocorreu durante momentos de lazer, e apenas 5\% dos animais agressores foram considerados por suas vítimas ou responsáveis como potencialmente doentes, devido à alteração de comportamento. No período de estudo, verificouse ainda a ausência da informação sobre este aspecto em $33,4 \%$ dos casos. Este fato foi observado em 26\% dos casos nos Estados Unidos (Center..., 2003).

Em relação às ocupações do paciente, observouse que os estudantes e as profissões que tradicionalmente ligam o paciente ao lar estão entre as principais ocorrências de atendimento antirrábico, o que também foi observado por Miranda et al. (2004), e somente 3,24\% das ocorrências relacionaram-se com trabalhadores rurais. No presente estudo, verificou-se que apenas em $29 \%$ dos casos os pacientes estavam relacionados a uma ocupação, diferentemente dos Estados Unidos, onde em $74 \%$ dos casos relacionam-se os pacientes a uma ocupação definida (Center..., 2003).

Durante o período de estudo, houve mudança do tipo de vacina utilizada e do número de doses necessárias para o tratamento antirrábico. A vacina utilizada até 2002 era a FuenzalidaPalacios modificada, substituída pela vacina de cultivo celular, cujo tratamento utiliza-se de duas a cinco doses para as mesmas indicações de tratamento e com menor risco de eventos adversos graves (Brito e Chamone, 2002). Com a mudança da vacina utilizada, houve um aumento no número de pacientes tratados nas áreas de baixo e médio risco para raiva. Por outro lado, o inverso ocorreu nas áreas de alto risco, como pode ser observado na Fig. 2.

A taxa geral de abandono do tratamento pelos pacientes foi de $9 \%$ e foi diferenciada entre as diversas DADs, considerando-se os dados da ZOO. No Brasil a taxa geral de abandono é de 13\% (Ministério..., 2002), e em Belo Horizonte é de 24,7\%, segundo Maurelli (2001).

A avaliação da ficha de atendimento do SINAN demonstrou que apenas os campos considerados obrigatórios para entrada da notificação estão completamente preenchidos. Os campos considerados importantes para a prescrição médica estavam, em grande parte, em branco. Essa negligência também foi verificada por Garcia et al. (1999), em São Paulo, e por Carvalho et al. (2002), no Paraná. A análise exploratória das fichas não permitiu verificar se houve erro de prescrição, já que não existe nas fichas um campo destinado ao registro do esquema de tratamento adotado. Igualmente, observou-se ainda a impossibilidade de rastreamento de animais agressores a partir dos registros, tampouco se houve a adoção de medidas como a lavagem do ferimento com água e sabão de acordo com a recomendação da (World..., 2001). 


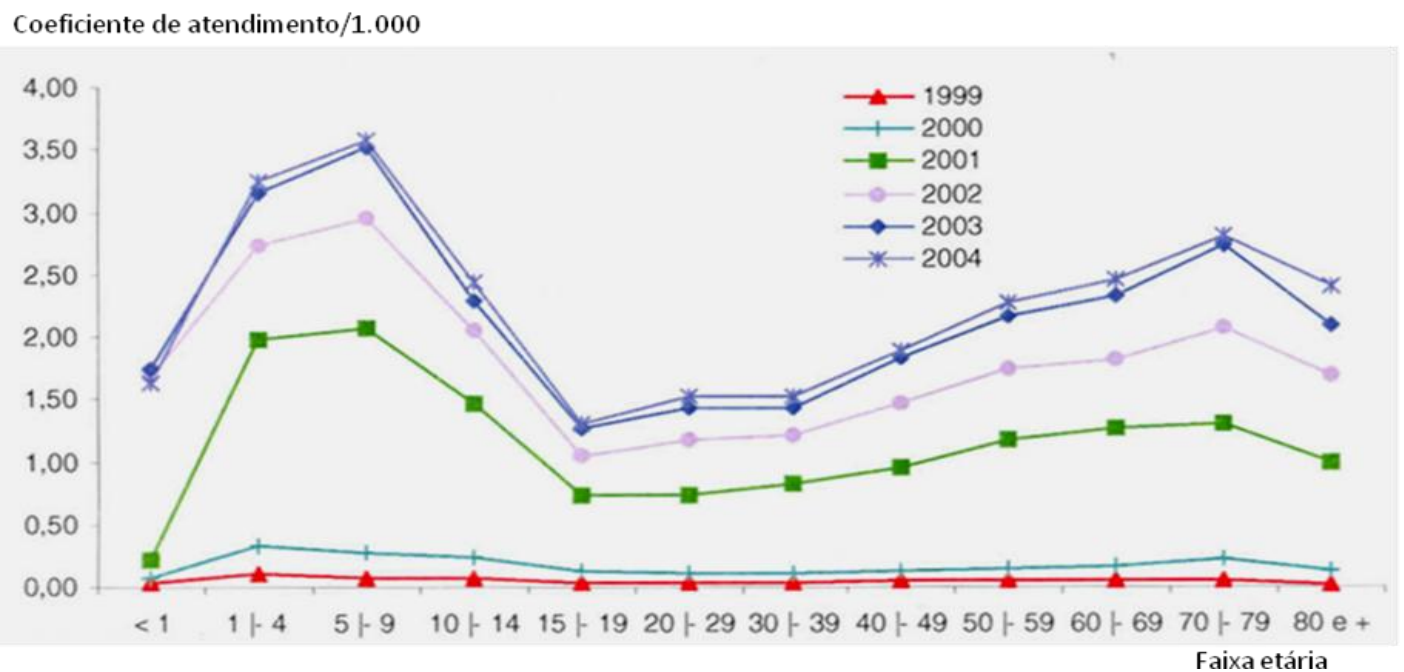

Figura 1. Coeficientes de atendimento antirrábico humano notificados no SINAN por faixa etária, Minas Gerais, 1999 a 2004.

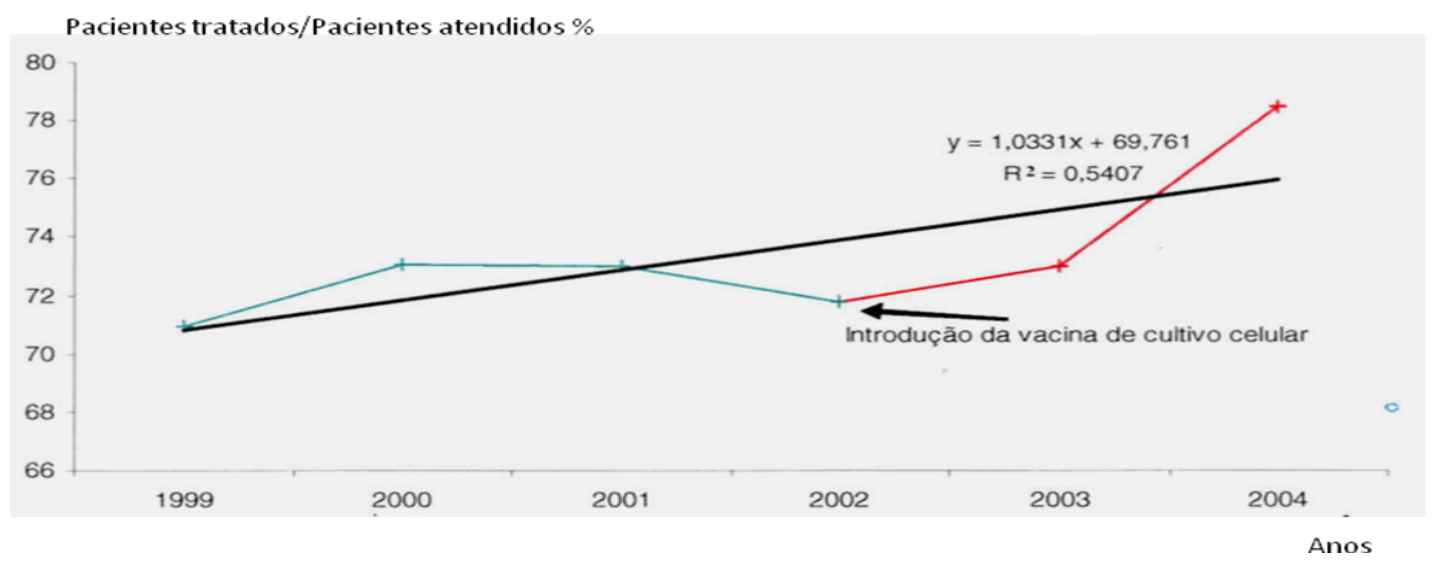

Figura 2. Percentual de pacientes tratados em relação aos pacientes atendidos observando-se a mudança no tipo de vacina utilizada, Minas Gerais, 1999 a 2004.

De modo geral, a ficha é complexa, com demasiados campos, o que torna difícil o seu preenchimento na unidade de atendimento. Talvez, por essa razão, dados importantes não são registrados, tais como: tratamento anterior, datas, códigos do agravo (CID 10) e busca ativa de pacientes faltosos. Por outro lado, não existem campos específicos para a identificação do responsável pelo tratamento, que é de responsabilidade médica, como relatado por Mateus e Moreira Filho (2005); para a evolução do paciente; sobre o animal agressor, seu estado vacinal, endereço da observação e veterinário responsável, como sugerido por Mazzoni et al. (2001). Igualmente, não existe um campo para relatar as agressões por herbívoros, relevantes na situação epidemiológica de Minas Gerais (Silva et al., 2001).

Durante o período de estudo, ocorreram cinco óbitos por raiva humana, dos quais três foram transmitidos por quirópteros, em áreas de médio e baixo risco para raiva humana transmitida por cão. A importância dos quirópteros na transmissão da raiva a seres humanos está relatada por France (2002). Nos casos restantes, o animal transmissor foi o cão, por mordedura canina, e ocorreram em área de alto risco para raiva.

No período de estudo, foram registrados pelo SIH 13 casos de internação por raiva humana, 
notificados e confirmados, CID A82, não coincidentes, entretanto, com os casos registrados no SINAN, tampouco no SIM. Esse desencontro de comunicação sugere inconsistência e falta de interação das informações entre os bancos de dados dos sistemas de notificação.

Ao se verificarem os dados das SIHs no banco de dados do SIM, foram detectadas, no período, 1.030 internações em razão de agressões por mamíferos (CID W54 e CID W55), sendo 427 internações devido a agressões por cães. Observou-se que o maior número de internações ocorreu na faixa etária de um a 10 anos, porém as pessoas idosas ficaram mais tempo (dias) internadas. Vale salientar que não houve suspeitos de raiva em nenhuma destas internações. Na Fig. 3, pode-se observar a distribuição espacial das internações devido à mordedura ou ao golpe proferido por cão. Esses agravos não são considerados problemas de saúde pública como causa externa pelo Sistema Único de Saúde no Brasil, exceto no tocante ao atendimento antirrábico. Nos EUA, essa questão já vem sendo tratada como agravo passível de controle e problema de saúde coletiva, segundo CDC (Center..., 2004).

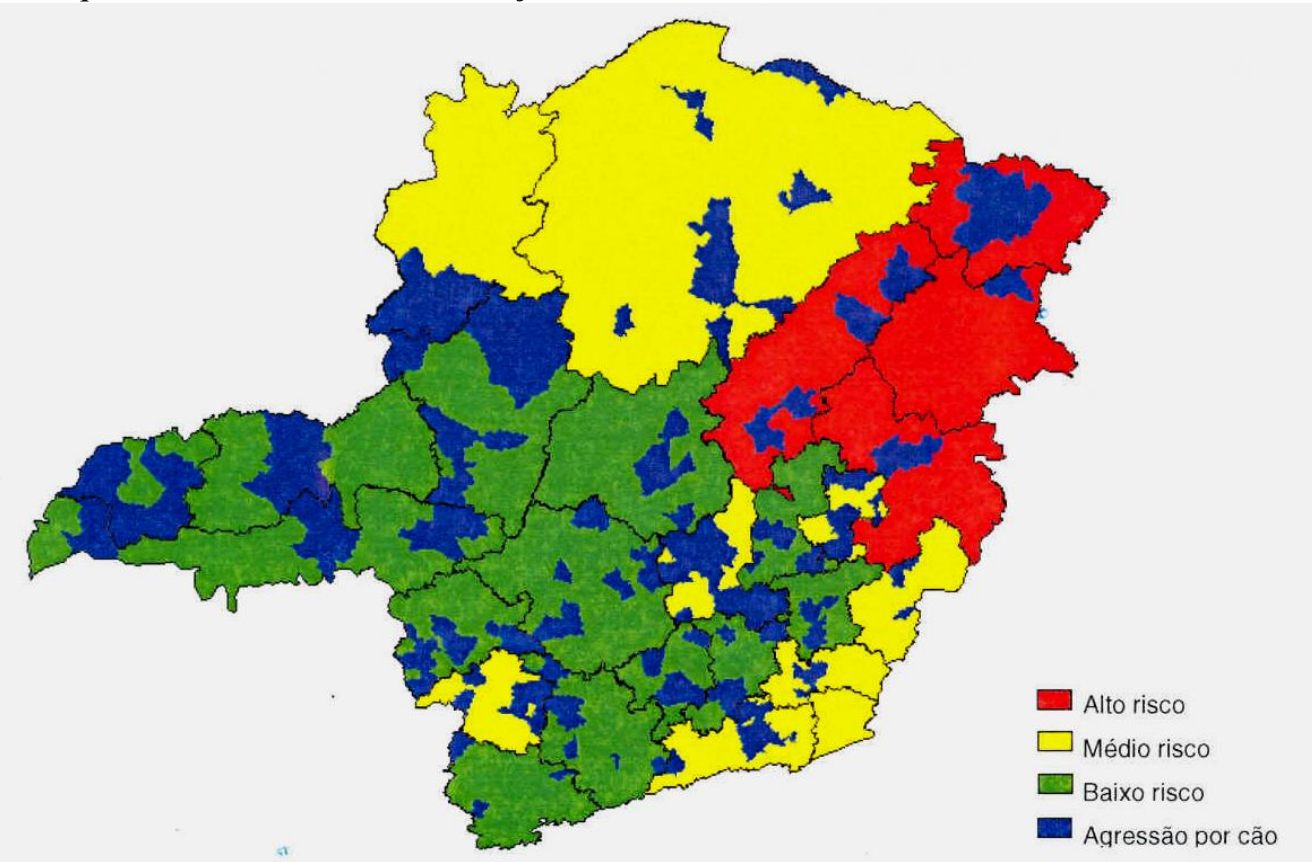

Figura 3. Internações devido às agressões por cães, nas áreas de risco da raiva humana transmitida por cão, Minas Gerais, 1999 a 2004.

\section{CONCLUSÕES}

A mordedura canina ainda é o principal agravo que leva o paciente a procurar o atendimento antirrábico humano. O perfil do paciente é estudante masculino, menor de 14 anos, residente em área urbana de baixo risco para raiva humana transmitida por cão, com mordedura única nos membros, provocada por cão sadio e observável. O tratamento é, de modo geral, excessivo, e nas áreas de baixo e médio risco, muitas vezes, desnecessário. Nas áreas de alto risco, verificou-se uma diminuição da demanda da indicação de tratamento, apesar da ocorrência, nessas áreas, de casos de raiva humana transmitida por cão. Os programas de profilaxia da raiva, SINAN, SIM,
SIH, PNI e ZOO, possuem bases isoladas, não atuando como rede de informação. Há subnotificações tanto por município silencioso quanto por informações incompletas ou distorcidas nos registros dos casos. $\mathrm{O}$ sistema de informação não oferece a confiabilidade necessária ao médico responsável para contraindicar a administração do imunobiológico, tampouco possibilita a interconsulta com o veterinário. A ausência de comunicação adequada e ágil entre ambos os profissionais propicia um exagero na prescrição e falhas na vigilância e no controle do agravo. Esse estudo pode subsidiar adequações no Programa de Profilaxia da Raiva em Minas Gerais, considerando-se o risco epidemiológico diferenciado. 


\section{REFERÊNCIAS}

AMATO NETO, V.; COSTA, W.A. Doenças Infecciosas em Pediatria In: FREIRE, L.M.S; TONELLI. E. Raiva Humana. 2nd. ed. Rio de Janeiro: Medsis, 2000, cap. 77, p.1165-1191.

ARAUJO, F.A. A situação da raiva no Brasil. In: SEMINÁRIO INTERNACIONAL DA RAIVA, 2000. São Paulo. Anais... São Paulo: Instituto Pasteur, p.22, 2000.

BELOTTO, A.J. Raiva canina - vigilância epidemiológica e caracterização de áreas de risco. In: SEMINÁRIO INTERNACIONAL DA RAIVA, 1., 2000, São Paulo. Anais... São Paulo: Instituto Pasteur, p.36-37, 2000.

BRITO, M.G.; CHAMONE, T.L. Ações de controle da raiva canina e felina no Estado de Minas Gerais 1999-2002. Boletim Epidemiológico da Superintendência de Epidemiologia, Secretaria de Estado da Saúde de Minas Gerais. Ano 6, n.4, p.1-3, 2002.

CARVALHO, W.O.; SOARES, D.F.P.P.S; FRANCESDRI, C.S. Características do atendimento prestado pelo serviço de profilaxia de Raiva Humana na rede municipal de saúde de Maringá-Paraná, no ano de 1997. Informe Epid. do SUS. v.11, p.25-35, 2002.

CENTER of disease control and prevention, CDC. National Center for Injury Prevention: National dog bite prevention week. 2004. Disponível em: <http://www.cdc.gov/ncipc>. Acessado em: 04 mar. 2005.

CENTER of disease control and prevention, CDC. Nonfatal Dog Bite Related Injuries Treated in Hospital Emergency Departments United. MMWRWeekly July 4, 2003 / v.52, n.26, p.605-610. 2003. Disponível em: <http://www.cdc.gov/mmwr/>. Acessado em: 04 mar. 2005.

DEAN, A.G.; DEAN, J.A.; BURTON, A.H. et al. EPIINFO: A word processing date base and statistics program for epidemiology on microcomputer. Georgia: USD, 1995. (Version 6.04b).

FRANCE, Epidemiologie et prophylaxie de la rage humaine en France, 2002. Disponível em <www.pasteur.fr/recherche/rage/bull2002.html>. Acessado em: 28 fev. 2009.

GARCIA, R.C.M.; VASCONCELOS, S.A.; SAKAMOTO, S.M. et al. Análise do Tratamento Antirrábico Humano pós-exposição em região da grande São Paulo, Brasil. Rev. Saúde Publ., v.33, p.295-301, 1999.
INSTITUTO Brasileiro de Geografia e Estatística (IBGE). 2000. In: Cidades - Informações Gerais. Disponível em: http://www.ibge.gov.br/cidadesat/ topwindow.htm?1 Acessado em: 25 fev. 2005.

MATEUS, J.A; MOREIRA FILHO, M.A. Prevenção da Raiva Humana, In: LEÃO; E.; CORREIA, E.; MOTTA, J. et al. Pediatria Ambulatorial, 4.ed. Belo Horizonte: Coopmed, 2005. cap.12, 2005, p.125-133.

MAURELLI, M.M. Características do tratamento antirrábico humano em Belo Horizonte, de 19902000. 2001. 84f. Dissertação (Mestrado em Medicina Veterinária) Escola de Veterinária, Universidade Federal de Minas Gerais, Belo Horizonte.

MAZZONI, M.; MARROCOS, S.; BERNARDES, D.F.R. Avaliação da conduta para profilaxia da Raiva pós- exposição na DRS de Varginha, Minas Gerais. 2001, 36f. Monografia (Especialização em Vigilância em Serviço) - Faculdade de Medicina, Universidade federal de Minas Gerais.

MINISTÉRIO da Saúde, Fundação Nacional de Saúde: Normas técnicas de tratamento profilático antirrábico humano. Ed Brasília: Fundação Nacional de Saúde, 2002.

MIRANDA, C.F.J.; SILVA, J.A.; MOREIRA, E.C. et al. Perfil do usuário do Serviço de Profilaxia da Raiva no Centro de Saúde Carlos Chagas, Belo Horizonte, Minas Gerais. Boletim Epidemiológico Eletrônico da Superintendência de Epidemiologia, Secretaria de Estado da Saúde de Minas Gerais, Minas Gerais: ano 1, n.2. 2004. Disponível em: <http://www.saude. mg.gov.br>. Acessado em: 08 fev. 2005.

MIRANDA, C.F.J.; SILVA, J.A.; MOREIRA, E.C. Raiva Humana transmitida por cães: área de risco em Minas Gerais, Brasil, 1991-1999. Cad. Saúde Pública, v.19, p.91-99, 2003.

MOREIRA, E.C.; GONTIJO, M.T.; CASTRO, A. et al. Aspectos epidemiológicos del tratamiento antirabico humano em Belo Horizonte, Minas Gerais, Brasil. Bol. Of. Sanit. Panam., v.80, p.38-44, 1976.

SAMPAIO, I.B.M. Estatística à experimentação animal. Belo Horizonte: Fundação de Estudo e Pesquisa em Medicina Veterinária e Zootecnia. 1998. 221p.

SILVA, J.A.; MOREIRA, E.C.; HADDAD, J.P.A. et $a l$. Distribuição Temporal e Espacial da Raiva Bovina em Minas Gerais, 1976 a 1997. Arq. Bras. Med. Vet. Zootec., v. 53, p.263-272, 2001.

WORLD health organization-WHO. Rabies, Fact Sheet n. 99 Revised June 2001. Disponível em: <http://www.who.int/mediacentre/factsheets/fs099/en>. Acessado em: 28 fev. 2009. 